\section{The Lanindar test: a method of evaluating patient suitability for cataract surgery using assisted topical anaesthesia}

${ }^{1}$ Ophthalmic Surgery Centre Chatswood, New South Wales, Australia

\section{${ }^{2}$ Department of Ophthalmology, Prince of Wales Hospital, Randwick, New South Wales, Australia \\ ${ }^{3}$ Vision CRC, School of Optometry and Vision Science, University of New South Wales, Sydney, Australia}

Correspondence: IC Francis, Chatswood Grove Eye Clinic, Suite 12, 12-14 Malvern Avenue, Chatswood, New South Wales 2067, Australia Tel: 6129411 3277; Fax: 61294131839 E-mail: iancfrancis@ gmail.com

Received: 4 May 2007 Accepted in revised form: 5 November 2007: Published online: 18 January 2008

\begin{abstract}
Aim To evaluate an office-based Lanindar (light and nociceptive interaction noting distress and response) test to assist in the assessment of patient suitability for assisted topical anaesthesia (ATA) during phacoemulsification.
\end{abstract}

Methods The Lanindar test was carried out at the preoperative assessment of 716 consecutive patients in the office of one of the authors (ICF). A standard desk lamp was shone in each patient's eye after pupillary dilation, while simultaneously elevating the upper eyelid digitally. A negative test indicated patient hypersensitivity to the light and aversion to digital pressure on the upper eyelid. A positive test was indicated by the patient feeling comfort and lack of blepharospasm and withdrawal in response to the light and digital pressure. $\chi^{2}$ and Fisher's exact tests were used to assess the association between Lanindar results and suitability for ATA. The positive predictive value and specificity of the test as an indicator of patient suitability for ATA were calculated. Visual acuities at 1 and 4 weeks post-operative periods were compared between the ATA and ALA/GA (assisted local anaesthesia/general anaesthesia) group of patients.

Results About $86.7 \%$ were Lanindar positive and $98.9 \%$ of these patients tolerated ATA. $\chi^{2}$ and Fisher's exact tests demonstrated a significant association of a positive Lanindar test with successful ATA $\left(\chi^{2}=660, P<0.001\right.$, Fisher's: $P<\mathbf{0 . 0 0 1})$. The positive predictive value and specificity of the test were $98 \%(95 \%$ $\mathrm{CI}=\mathbf{9 8 . 0 4 - 9 9 . 7 \% )}$ and $93.14 \%(95 \% \mathrm{CI}=\mathbf{8 8 . 2 3}$ $\mathbf{9 8 . 0 4 \% ) , ~ r e s p e c t i v e l y . ~ V i s u a l ~ a c u i t y ~ o u t c o m e s ~}$ were similar in the ATA and ALA/GA groups.
EC Figueira', NS Sharma², J-L Ooi², K Masselos², KJY Lee ${ }^{2}$, ML Rosenberg' ${ }^{2}$ IC Francis $^{1,2}$, SL Alexander ${ }^{1}$, NI Ferch ${ }^{1}$ and F Stapleton ${ }^{3}$
Conclusion The Lanindar is a simple, highly specific, office-based test to determine patient suitability for phacoemulsification under ATA. Eye (2009) 23, 284-289; doi:10.1038/sj.eye.6703080; published online 18 January 2008

Keywords: Lanindar test; topical anaesthesia; phacoemulsification; specificity

\section{Introduction}

Phacoemulsification cataract surgery is one of the most common surgical procedures carried out today. ${ }^{1}$ The most appropriate anaesthetic modality should be selected for every patient. There are three main forms of anaesthesia for cataract surgery. First, general anaesthesia (GA) is rarely used. Second, peribulbar/ retrobulbar/subtenon's assisted local anaesthesia (ALA) ${ }^{2}$ is being used less frequently. Third, with the introduction of clear corneal phacoemulsification surgery, assisted topical anaesthesia (ATA) is becoming more popular. By ATA, we refer to anaesthesia support along with an anaesthetist administering sedation where required as well as monitoring the patient. ${ }^{3}$

On reading the ophthalmological literature, one regularly comes across the statement that a certain patient was not suitable for topical anaesthesia. ${ }^{4}$ In spite of this, to our knowledge, no criteria have been offered to determine a patient's suitability for ATA in comparison with ALA.

The first successful use of topical anaesthesia (5\% cocaine) for cataract surgery was reported by $\mathrm{Knapp}^{5}$ in the year 1884 . Topical anaesthesia 
for cataract surgery was also used in the year 1910 by Hirschberg, ${ }^{6}$ who used a $2 \%$ cocaine chloride solution as topical anaesthesia for thousands of cataract procedures and reported only advantages. Smith, ${ }^{7}$ in the 1980s, performed cataract surgery under topical anaesthesia with supplementary subconjunctival injection.

ATA may ultimately prove to be the safest mode of anaesthesia as it avoids the systemic risks of GA and the risk of trauma to the orbital contents and of orbital swelling, that accompany regional blocks including that of subtenon. ${ }^{8}$ For many patients and surgeons, ATA fulfils all the goals of anaesthesia in cataract surgery. The disadvantages of ATA are increased ocular motility, the requirement for patient cooperation, and the fact that intravenous sedation may prove to be necessary. ${ }^{9}$ Patients during cataract surgery have recorded that manipulation (pressure-like pain) and the microscope light (glare) bothered them. ${ }^{10}$

In this prospective consecutive study, we describe the use of the Lanindar (light and nociceptive interaction noting distress and response) test in evaluating patient suitability for ATA.

\section{Methods}

A total of 716 consecutive cases scheduled for phacoemulsification surgery were prospectively studied. As part of the routine preoperative assessment, the Lanindar test was performed on each patient in the office. The study was carried out according to the tenets of the Declaration of Helsinki, and ethics approval was obtained by the Ethics panel of the University of New South Wales, Australia, for the collection of the data (UNSW HREA approval ref. no. 074008).

The Lanindar test evaluates patient tolerance to the bright light of the office desk lamp and tolerance to the examiner's digital pressure on elevating the eyelid of the eye to be operated on. The Lanindar test was performed in the surgeon's office (ICF) at the preoperative cataract surgery assessment. All patients were adapted to the background illumination of the office for at least $10 \mathrm{~min}$. The patients pupils at this stage have already been dilated. The light was held $25 \mathrm{~cm}$ from the patient's face. The illumination of the light source for the test in the office and of the operating microscope was measured using a calibrated Hagner Digital Luxmeter, model EC1 (Hagner ${ }^{\circledR}$, Solna, Sweden). The surrounding illuminance of the examination room at the level of the patients' eyes and the illumination of the operating theatre was measured using the same instrument. The luminance of the background illuminating light and testing light source (desk lamp) of the test conditions in the office was closely simulated to the luminance of the background illuminating source and operating microscopic light (in the operating theatre). In the same way as the Lanindar test was performed on eyes with mydriatic pupils after the patient had been light-adapted for at least $15 \mathrm{~min}$, the operated patients were exposed to the operating microscope light after background light adaptation.

The patient's response was sometimes manifested by involuntary eyelid closure/blepharospastic-like closure of the eyelids or withdrawal of head and eyes from the light source. This was considered a negative Lanindar response (Figure 1). On the other hand, the patient generally demonstrated indifference to the light stimulus. This was regarded as a positive Lanindar response (Figure 1) and was considered as predicting suitability for ATA.

Prospective descriptive data for the 716 patients were recorded. In particular, patient age, gender, type of Lanindar response, and type of anaesthesia used (GA, ALA, or ATA) were assessed.

Prior to surgery, either Midazolam (1-3 mg) or Alfentanyl (100-300 $\mu \mathrm{g}$ ) was used to provide appropriate sedation and anxiolysis. The dose of these medications
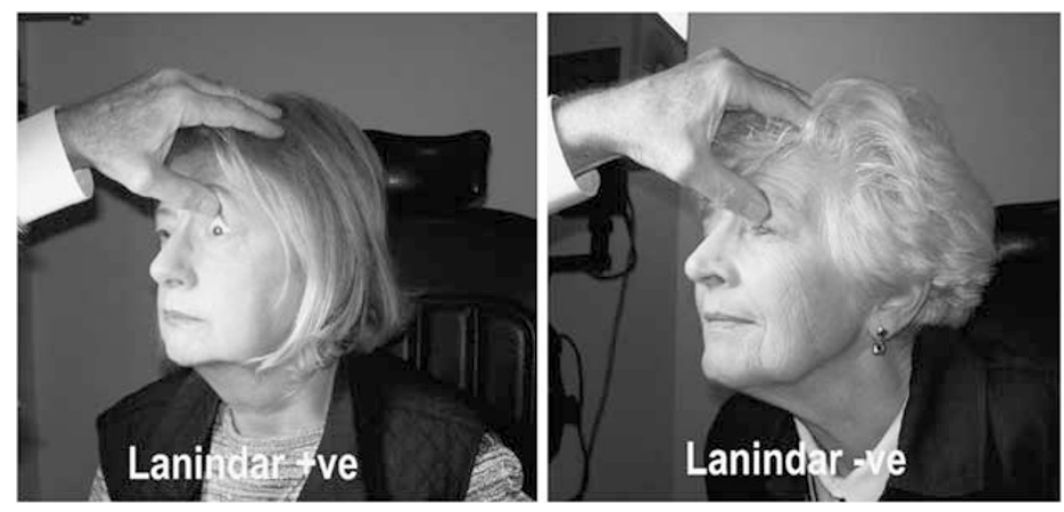

Figure 1 This figure indicates the Lanindar test: right panel—Lanindar-positive response; left panel—Lanindar-negative response. 
was titrated to each patient such that they were awake and responsive to the surgeon's instructions during the surgery and were orientated to the operating theatre environment. The patients then underwent clear corneal incision phacoemulsification surgery.

A contingency $2 \times 2$ table analysis of the data was evaluated for the following groups using $\chi^{2}$ and Fisher's exact tests. These comprised the cohort that had a positive Lanindar and tolerated ATA; the cohort that had a positive Lanindar in the office but was converted to ALA or GA; the cohort that had a negative Lanindar in the office and required ALA; and the cohort that was Lanindar negative in the office but for whom it was decided to employ ATA (0 patients). A $\chi^{2}$-test and Fisher's exact test analysis were performed to determine an association between a positive Lanindar response and successful phacoemulsification surgery using ATA. Specificity and positive and negative predictive values for the test were also evaluated from the $2 \times 2$ contingency table. The sensation of discomfort and glare induced by the office lamp used for the test and the operating microscope within their surroundings was calculated. Ferguson and Richards, ${ }^{11}$ in their review on lighting technology and glare comfort, described that the degree to which glare is experienced largely depends on the following four factors: the luminance of the glare source $L s$, the subtense of the source at the eye $\omega$, the general background luminance about the source $L B$, and the angle between the line of sight and the direction of the source $\theta$. Increases in $L s$ and $\omega$ worsen the glare sensation, while increases in $L B$ and $\theta$ lessen it. With the assumption that the angle between the line of sight of the patient and the direction of light source are almost coaxial (roughly $1^{\circ}$ ), the calculated induced discomfort-glare constant index in the subject's eyes by the office halogen lamp at $25 \mathrm{~cm}$ was 146.028 (glare constant index). Similarly, the induced discomfortglare constant index in the subjects' eyes by the operating microscope light source was calculated as 110.101 using the formula below as described by Ferguson and Richards. ${ }^{11}$

Objective glare disability index $=\operatorname{Ls} \omega^{0.8} / \mathrm{LB} \theta^{2}$, where $L s$ is the luminance of the glare source, $\omega$ is the subtense of the source in steradians, $L B$ is the general background, and $\theta$ is the angle between the line of sight and the direction of the source. ${ }^{11}$

The best spectacle-corrected visual acuity (BSCVA) in patients operated using ATA or ALA/GA was compared at the preoperative visit, 1 and 4 weeks following the surgery. These were compared to assess if the visual outcome of cataract surgery was similar or different between the two groups. Incidences of complications were compared between the two groups.

\section{Results}

The patients' ages ranged from 34 to 98 years (mean 73.7). There were 268 males (37.4\%) and 448 females $(62.6 \%)$.

The Lanindar test was positive in 621 cases $(86.7 \%)$. Seven of the $621(1.13 \%)$ Lanindar-positive cases were converted to an ALA or GA during the surgery, necessitating redraping and re-anaesthetising the patient (Figure 2).

The mean age for Lanindar-positive patients was 73.58 years (range $=34-98$ years) and for Lanindar-negative patients was 73.96 years (range $45-98$ years) $(P=0.39$ value for $t$-test comparing age difference in patients who were Lanindar positive and negative). Two hundred and twenty-six $(84.3 \%)$ of the total 268 male patients were Lanindar positive and $395(88.2 \%)$ of the total 448 female patients were Lanindar positive. The $\chi^{2}$ association of gender with Lanindar test results revealed a non-statistically significant value of $2.15(P=0.143)$, suggesting that the Lanindar results are not influenced by gender (Table 1). There was no observable pattern for patients who were converted from ATA to ALA or GA.

$\chi^{2}$ analysis of the contingency table revealed a strong association between a positive Lanindar test and a successful ATA procedure (Pearson's $\chi^{2}$-value 660, $P<0.0001$ ) (see Table 1a and b). To clearly demonstrate an association between the variables, the Fisher's exact test also revealed a strong association $(P<0.001)$ between

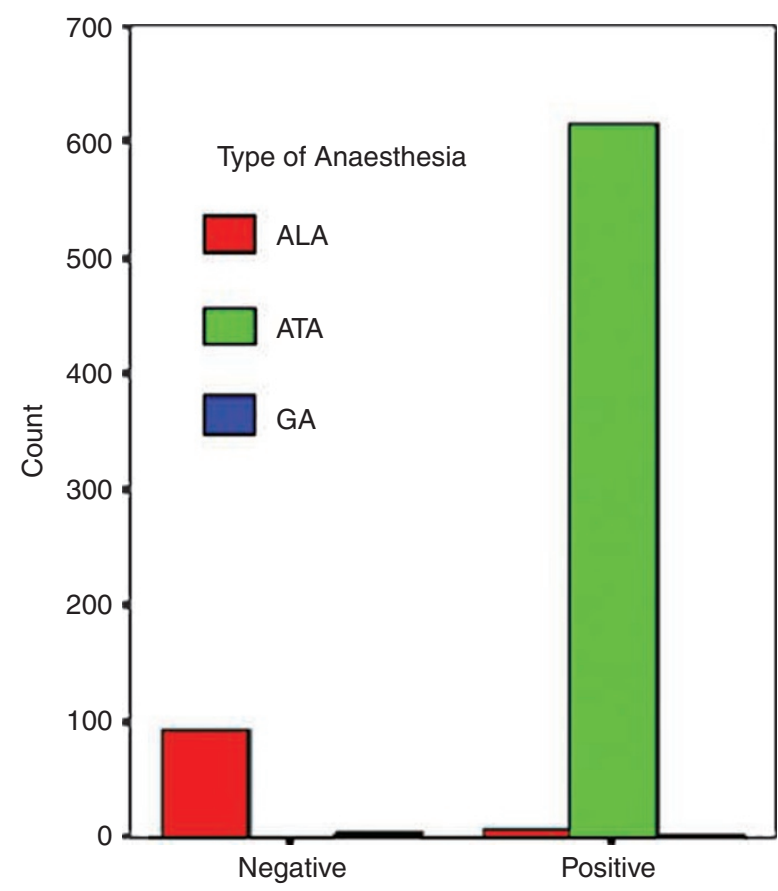

Figure 2 This bar chart describes the results of the Lanindar test and the type of anaesthesia used for the surgery. 
Table $12 \times 2$ Contingency analysis association of Lanindar test results and type of anaesthesia

\begin{tabular}{|c|c|c|c|}
\hline \multicolumn{4}{|c|}{ (a) Lanindar test results and type of anaesthesia used displayed in the $2 \times 2$ contingency table } \\
\hline & $A L A(G A)$ & ATA & Total \\
\hline Lanindar-negative test & $92(3)$ & 0 & 95 \\
\hline Lanindar-positive test & $6(1)$ & 614 & 621 \\
\hline Total & $98(4)$ & 614 & 716 \\
\hline \multicolumn{4}{|c|}{ (b) $\chi^{2}$-test results from the above $2 \times 2$ contingency analysis for the 716 cases } \\
\hline & Value & Degree of freedom & Significance (two-sided) \\
\hline Pearson $\chi^{2}$-value & 660.536 & 2 & $P<0.0001$ \\
\hline Likelihood ratio & 510.918 & 2 & $P<0.0001$ \\
\hline \multicolumn{4}{|c|}{ (c) Fisher's test results for the same $2 \times 2$ contingency analysis for the 716 cases } \\
\hline \multicolumn{4}{|c|}{ Fisher's exact test } \\
\hline \multicolumn{4}{|l|}{$P$-value $P<0.0001$ (two-sided) } \\
\hline Odds ratio $=15650(95 \% \mathrm{CI}=885.9-276400)$ & & & \\
\hline
\end{tabular}

Table 2 Sensitivity, specificity, and predictive values of the Lanindar test

\begin{tabular}{lrrr}
\hline & $($ ATA) & (ALA/GA) & Total \\
\hline Test positive & 614 & 7 & 621 \\
Test negative & 0 & 95 & 95 \\
Total & 614 & 102 & 716
\end{tabular}

\begin{tabular}{lcrr} 
& Proportion & CI \\
\cline { 2 - 3 } & Estimate & Lower & Upper \\
\cline { 2 - 3 } Sensitivity of Lanindar test & 1.00 & 1.00 & 1.00 \\
Specificity of Lanindar test & 0.93 & 0.88 & 0.98 \\
Likelihood ratio of Lanindar-positive test & 14.57 & 7.13 & 29.78 \\
Probability of Lanindar-positive patients suitable for ATA & 0.86 & 0.83 & 0.88 \\
Predicted-positive value of the Lanindar test & 0.988 & 0.98 & 0.997 \\
Predictive-negative value of the Lanindar test & 1.00 & 1.00 & 1.00 \\
Overall accuracy & 0.99 & 0.98 & 0.997 \\
\hline
\end{tabular}

the positive Lanindar test and successful completion of small incision phacoemulsification cataract surgery using ATA. The calculated odds ratio of the association revealed a value of 15650 (95\% CI $=885.9-276400)$.

Specificity values were calculated in a $2 \times 2$ contingency table analysis as shown in Table 2.

The Lanindar test used to predict the suitability of patients for ATA has a high specificity of $93.14 \%(95 \%$ $\mathrm{CI}=88.2-98.04 \%$ ). The positive predictive value for the Lanindar test to predict suitability of the patient for ATA is estimated as $98.97 \%$ (95\% CI $=98.04-99.7 \%)$. The glare disability induced (measured as glare constant units) by the office halogen lamp at $25 \mathrm{~cm}$ was calculated as 146.028 Glare constant units and that induced by the operating microscope light source was calculated as
110.101 Glare constant units, when measured objectively using a photometer and described calculations (see the Methods section). Ferguson and Richards ${ }^{11}$ suggested that these constant values can be compared to some standard levels (eg 600 as intolerable and 150 as uncomfortable).

The illumination of the light source for the test in the office measured using a calibrated Hagner Digital Luxmeter, model EC1 (Hagner ${ }^{\circledR}$, Solna, Sweden) was found to be 1119 Lux (average of three readings) at $25 \mathrm{~cm}$. The surrounding illuminance of the examination room at the level of the patient's eyes was 121 Lux (average of three readings) when measured by the same instrument. The illumination of the test light source was not very different from the illumination source of the operating 
Table 3 Comparison of visual acuity outcomes in the ATA and ALA/GA group of patients (SD)

\begin{tabular}{lccr}
\hline & ATA surgery & ALA surgery & Student's t-test \\
\hline Pre op BSCVA & 0.54 (SD 0.42) & 0.53 (SD 0.25) & $P>0.05$ \\
Post-operative 1 week BSCVA & 1.14 (SD 0.31) & 1.13 (SD 0.34) & $P>0.05$ \\
Post-operative 4 weeks BSCVA & 1.31 (SD 0.29) & 1.31 (SD 0.32) & $P>0.05$ \\
\hline
\end{tabular}

microscope at the same distance of $25 \mathrm{~cm}$ (1300 Lux (average of three readings) at $25 \mathrm{~cm}$ on a background of an operating room illumination of 576 Lux (average of three readings)).

The BSCVA measured preoperatively and postoperatively at 1 and 4 weeks are summarised in Table 3 below. There was no statistical difference in BSCVA at the preoperative visit and post-operative 1 and 4 weeks follow-up.

The preoperative BSCVA and post-operative BSCVA at 1 and 4 weeks were similar in both groups (ATA and ALA/GA) (Student's $t$-test, $P>0.05)$. The only intraoperative complication observed in the entire cohort of patients was posterior capsular tears with or without vitreous loss. Posterior capsular tears occurred in 7/614 (1.14\%) patients who were operated using ATA. This was comparable to frequency of posterior capsular tears that occurred in 1/95 (1.05\%) patients who were operated using ALA.

\section{Discussion}

In the year 1993, Kershner et al ${ }^{12}$ evaluated 100 patients undergoing cataract surgery under topical anaesthesia and concluded that topical anaesthesia was safe, minimised surgical complications, and allowed rapid visual recovery. Patel et $a l,{ }^{4}$ in a study comparing topical and retrobulbar anaesthesia for cataract surgery, reported that light bothered $10 \%$ of cases who were operated under topical anaesthesia, mostly occurring during the initial part of the operation. We therefore used a unique photometric objective measurement of glare, closely simulated the surgical theatre environment to the environment in the examination office, by utilising a similarly bright office lamp in the Lanindar test procedure. Patel et $a l^{4}$ also suggested that, for topical anaesthesia, careful selection of the patients was essential for the smooth intraoperative and post-operative course of the surgery.

Therefore, any objective test in the preoperative evaluation of cataract patients to identify those who are suitable for ATA should be helpful. To date and to our knowledge, there have been no studies published indicating which patients are suitable for ALA vs ATA. Some ophthalmologists attempt to perform all their cataract surgery using ATA. Claoue ${ }^{13}$ reported that patients under topical anaesthesia described a pressure sensation during surgery. Due to the nature of topical anaesthesia, pressure and temperature sensation are experienced by the patient. Topical anaesthesia is typically described as analgesic but not anaesthetic. For ideal scientific confirmation, to determine if the Lanindar was strongly predictive of the suitability to topical anaesthesia, both positive and negative responders should have been enrolled for ATA. We felt, however, that it would have been unethical to expose a Lanindarnegative patient to ATA.

In our series, the Lanindar test allowed us to decide objectively whether or not to utilise ATA. Seven patients whom we predicted were suitable for ATA proved intolerant of ATA and had their mode of anaesthesia changed (see Table 1). Two of the seven patients did not tolerate the bright light of the dimmed operating microscope. One was an 87-year-old male and the other was a 71-year-old female. A third patient initially planned to be operated using ATA was finally operated using GA as she was coughing through her tracheostomy once prepped and the eyelid speculum had been inserted. The fourth and fifth patients were shifted to ALA due to poor pupillary dilatation. The remaining two patients were shifted from ATA to ALA due to excessive eye movement.

The specificity of the Lanindar test in identifying cases that were not suitable for ATA was $93.14 \%$ (95\% $\mathrm{CI}=88.23-98.04 \%)$. The sensitivity of the test could not be reliably determined due to the lack of a known accepted published standard in relation to patient suitability for ATA. To evaluate the sensitivity of the Lanindar test in the future, we would regard patients assessed as having a negative Lanindar test being operated on under ATA in an attempt to determine the need for their shifting to ALA as unethical. Second, there are no standard benchmarks or results of test to evaluate patient suitability to be operated under ATA, against which we can truly ascertain or compare our results with. Is this last sentence not repetitive compared with the second sentence in this paragraph? Furthermore, there are no studies currently available that provide guidelines, which indicate the rate of shifting from ATA to ALA. Similar visual acuity outcomes after cataract 
surgery using ATA or ALA/GA also suggested that there was no difference in the final outcome after cataract surgery in the two groups. In the ATA group of patients, there were 7 out of 614 cases $(1.15 \%)$ (posterior capsular tears) (five were implanted with a primary ciliary sulcus intraocular lens, two had vitrectomies and then later had sulcus-placed secondary intraocular lens implantation). In the ALA group, there was a single case of vitreous loss after posterior capsular tear $(1 / 95=1.05 \%)$, in which a secondary IOL was implanted later.

Thus, the Lanindar test appears to be a simple and specific objective method of predicting in the office environment which patients may tolerate phacoemulsification surgery under ATA.

\section{References}

1 Schein OD, Katz J, Eric B, Tielsch JM. The value of routine preoperative medical testing before cataract surgery. $\mathrm{N} \mathrm{Engl}$ J Med 2000; 342: 168-175.

2 Francis IC, Schumacher RS, Haylen MJ. Assisted local anaesthesia for cataract surgery (ALACS). Aust NZ J Ophthalmol 1987; 15: 185-191.

3 McK Smiles JJ. A question of semantics. J Cataract Refract Surg 2006; 32: 907.
4 Patel BC, Burns TA, Crandall A, Shomaker ST, Pace NL, van Eerd A et al. A comparison of topical and retrobulbar anesthesia for cataract surgery. Ophthalmology 1996; 103: 1196-1203.

5 Knapp H. On cocaine and its use in ophthalmic and general surgery. Arch Ophthalmol 1884; 13: 402-448.

6 Hirschberg J. History of Ophthalmology, Volume 3, Part 1. Translated by Frederick C Blodi Verlag JP Wayenborgh: Bonn, Germany, 1982.

7 Smith R. Cataract extraction without retrobulbar anaesthetic injection. Br J Ophthalmol 1990; 74: 205-207.

8 Kumar CM, Dowd TC, Dodds C, Boyce R. Orbital swelling following peribulbar and sub-tenon's anesthesia. Eye 2004; 18: 418-420.

9 Navaleza JS. Choosing anesthesia for cataract surgery. Ophthalmol Clin North Am 2006; 19: 233-237.

10 Crandall AS, Zabriskie NA, Patel BC, Burns TA, Mamalis N, Malmquist-Carter LA et al. A comparison of patient comfort during cataract surgery with topical anaesthesia versus topical anesthesia and intracameral lidocaine. Ophthalmology 1999; 106: 60-66.

11 Ferguson HM, Richards MJ. A review of lighting technology. Review of Physics in Technology 1972; 3: 69-107.

12 Kershner RM. Topical anaesthesia for small incision selfsealing cataract surgery. A prospective evaluation of the first 100 patients. J Cataract Refract Surg 1993; 19: 290-292.

13 Claoue C. Topical anaesthesia for cataract surgery. Aust NZ J Ophthalmol 1997; 25: 265-268.

Supplementary Information accompanies the paper on Eye website (http://www.nature.com/eye) 